\title{
Investigation of the genetic background of Hungarian patients with nevoid basal-cell carcinoma syndrome
}

\author{
Dalma Márton ${ }^{1}$, Norbert Kiss ${ }^{1}$, Kende Lőrincz ${ }^{1}$, András Bánvölgyi ${ }^{1}$, \\ Nóra Gyöngyösi ${ }^{1}$, Márta Medvecz ${ }^{1}$ and Norbert Wikonkál ${ }^{1}$ \\ 1 Department of Dermatology, Semmelweis University, Budapest, Hungary
}

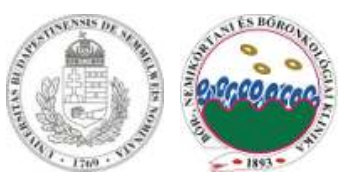

\section{Introduction}

Nevoid basal-cell carcinoma syndrome (NBCCS, OMIM:109400) is a rare genetic multisystem disorder. It is characterized by the occurrence of multiple basal cell carcinomas usually before 35 years of age, odontogenic keratocysts, plantar and palmar pits, ectopic intracranial calcification, most often of the falx cerebri and ophthalmic, neurological, skeletal and dental abnormalities [1]. The main underlying genetic abnormality for the disease is mutations in the PTCH gene or in other genes in the Hedgehog signaling pathway. Data from the literature show that heterozygous germline PTCH1 mutations are expected to be around $65 \%$ [2].

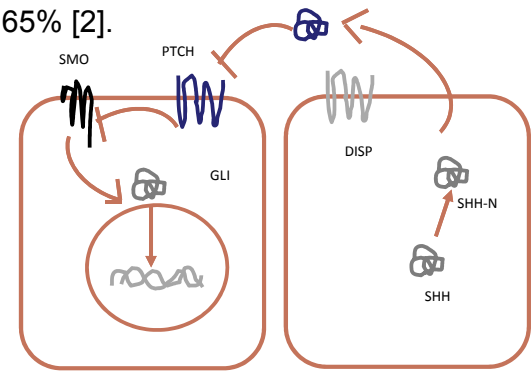

Figure 1. The Hedgehog signalling pathway

\section{Materials and methods}

Our research group set out to perform the DNA sequencing analysis of the PTCH1 gene of Hungarian NBCCS patients in order to identify genetic alterations. All study participants meeting clinical diagnostic criteria for familial or sporadic Gorlin-Goltz syndrome. We carried out the sequencing of the PTCH1 gene from 20 patients. We isolated DNA from the patients, and with the use of suitable primers, we amplified the 23 exons of the PTCH1 gene one by one utilizing PCR technique followed by automatic sequencing. We compared the acquired nucleotide sequences for both the reverse and the forward DNA strain to the normal variant found in the databases.

\section{References}

\section{Results}

Functional mutations in the PTCH1 were identified in only 7 patients. In 1 patient, it was a stop codon insertion, in 3 patients, frameshift mutations, while in further 3 patients single base mutations that resulted in amino acid change. The analysis of protein folding showed that the amino acid changes affect protein-protein interactions. This ratio (35\%) is significantly lower than we expected from the literature (50-75\%) [2].

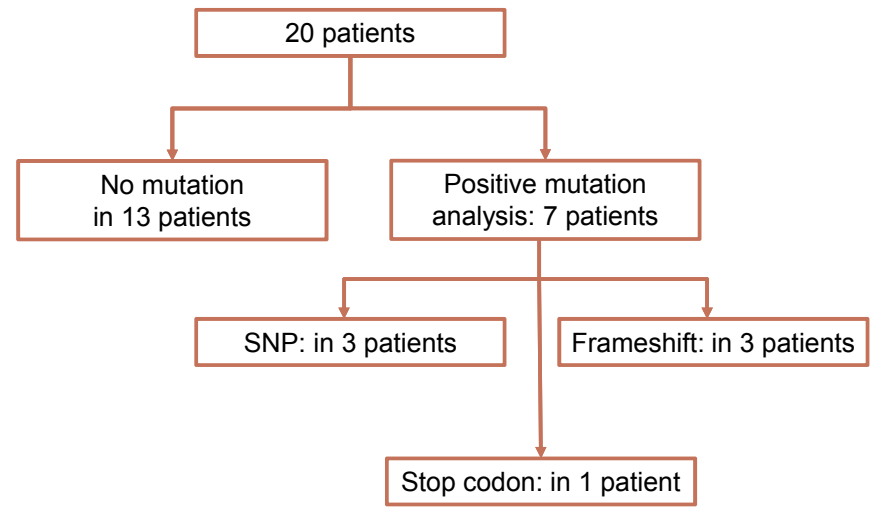

Figure 2. Mutation analysis was positive in the $35 \%$ of our patients with NBCCS. $75 \%$ of the patiens were wild type, in $5 \%$ we found a stop codon, $15 \%$ had a frameshift mutation, and $15 \%$ of the patients had a single nucleotide polymorphism.

\section{Future plans}

We plan to continue the experiments to investigate that in cases where mutations in the PTCH1 was not found what genes may be responsible for the disease.

We intend to increase the number of the patients involved, exclude the role of macro-deletions and also perform the sequencing of other parts of the PTCH gene (as promoter and intron regions) and or additional genes of the Hedgehog pathway (e.g. SMO, SUFI, GLI). The identification of the mutations responsible for the disease could support further evidence for the prenatal screening and counseling of NBCCS patients in the future.

1. Crawford KM, Kobayashi T. Nevoid basal cell carcinoma syndrome or multiple hereditary infundibulocystic basal cell carcinoma syndrome? Journal of the American Academy of Dermatology. 2004;51(6):989-95.2.

2. Fujii K. and Miyashita T. Gorlin syndrome (nevoid basal cell carcinoma syndrome): update and literature review. Pediatr Int, 2014;56(5):667-74. 